The Be Phenomenon in Early-Type Stars, IAU Colloquium 175

ASP Conference Series, Vol. 214, 2000

M. A. Smith, H. F. Henrichs, and J. Fabregat, eds.

\title{
59 Cygni: A Tilted $\phi$ Persei like System
}

Th. Rivinius

Landessternwarte Königstuhl, D-69117 Heidelberg, Germany

S. Štefl

Astronomical Institute, Academy of Sciences, CZ-25165 Ondřejov, Czech Republic

Abstract. The binarity of 59 Cygni was confirmed and orbital parameters from the radial velocity curve of the primary derived. The observed emission variability resembles the one of $\phi$ Per in quite some detail, suggesting a similar nature of the companion: a hot, compact star.

\section{Introduction}

59 Cygni (HR 8047, HD 200120, B1.5Ve) is for several reasons a famous and well observed Be star. Several investigators reported variability with a typical timescale of 28 to 29 days and suggested that the star is a spectroscopic binary (Barker 1983, Doazan et al. 1985 and 1989, Tarasov and Tuominen 1987). It has furthermore spatially resolved companions, being quadruple in total. The onset of a new Be phase in the late 70's made the star a primary target for IUE monitoring of the envelope formation. Soon after the emission re-appeared, the intensity ratio of the violet and red peaks $(V / R)$ of $\mathrm{H} \alpha$ started to vary with a quasiperiod of about 2 years. The variations ceased in the mid 80 's. Additionally, it is one of the few Be stars for which "spectacular variations" have been observed (Barker 1983, Hummel 1998). Up to now, however, neither reliable orbital parameters were published nor was an investigation about the nature of the secondary undertaken.

59 Cygni was observed from the Heidelberg and Calar Alto observatories (Table 1) as secondary target every other day in an ongoing search program for multiperiodic line profile variability of Be stars and its connection to outbursts (e.g. Stefl and Rivinius, Tubbesing et al., both this volume) using the Heros spectrograph (Kaufer, 1998).

\section{The Orbit of the Primary}

From our observations in 1998 (cf. Table 1) a regular pattern was well visible at first inspection of the data (Fig. 1, left). Especially from variations of photospheric lines, like He I 4471, it became apparent that the system is indeed a single-lined spectroscopic binary. The radial velocity (RV) of the He I 4471 line was measured in a first step interactively using a Gaussian. We con- 

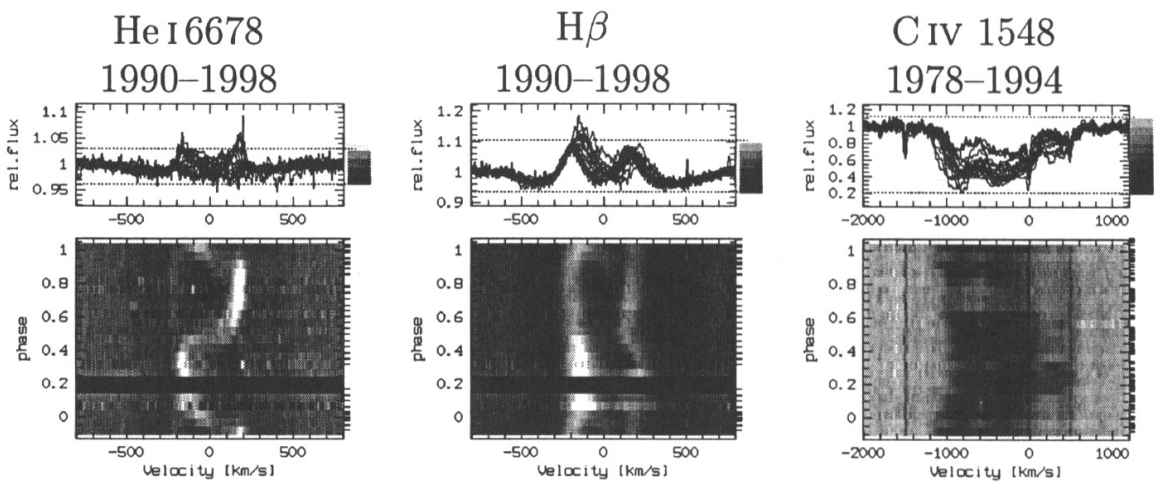

Figure 1. Phased dynamical spectra of $\mathrm{He}$ I 6678, $\mathrm{H} \beta$, and CIV 1548. In the Heros data (left two panels) the broad photospheric absorption component, the steady circumprimary disk, and the emission feature varying in antiphase wrt. the absorption can be seen, while the IUE data (right panel) demonstrates the long-term coherence of the period of 28.1702 day.

Table 1. Heros observing log of 59 Cyg

\begin{tabular}{cccc}
\multicolumn{2}{c}{ Observing } & $\begin{array}{c}\text { No. of } \\
\text { nights }\end{array}$ & $\begin{array}{c}\text { No. of } \\
\text { spectra }\end{array}$ \\
\hline 1990 July & LSW, $72 \mathrm{~cm}$ & 23 & 9 \\
1996 Oct. & LSW, 72 cm & 1 & 1 \\
1997 Sep. & LSW, 72 cm & 1 & 1 \\
1998 Aug./Sep. & Calar Alto, $1.23 \mathrm{~m}$ & 64 & 37
\end{tabular}

structed an RV-corrected mean profile for HeI 4471 . This profile was taken as a template for a cross-correlation analysis, yielding the final RV measurements. With this method the average velocity is by definition zero, so that no reliable systemic $(\gamma)$ velocity was derived. Due to aliasing, the HEROs data alone gives ambiguous periods. Using published data, i.e. the IUE spectra (184 in the short wavelength range from 1978 to 1994 , Fig. 1, right), we could fix the period to $28.1702 \pm 0.0014$ day. We used the VELOC (W. Schmutz) and FOTEL (P. Hadrava) codes to derive the orbital parameters from the RV measurements. Table 2 shows the orbital solution, Figure 2 the resulting RV curve. The Balmer emission morphology (Fig. 1, middle), as well as the absence of orbital light variations and phase-locked shell phases indicates that the system is viewed at an intermediate inclination angle.

\section{59 Cyg as $\phi$ Per-Like System}

The most peculiar features in the $\phi$ Per spectrum are the appearance and variability of emission components in the He I lines (Gies et al., 1992, Štefl et al., 


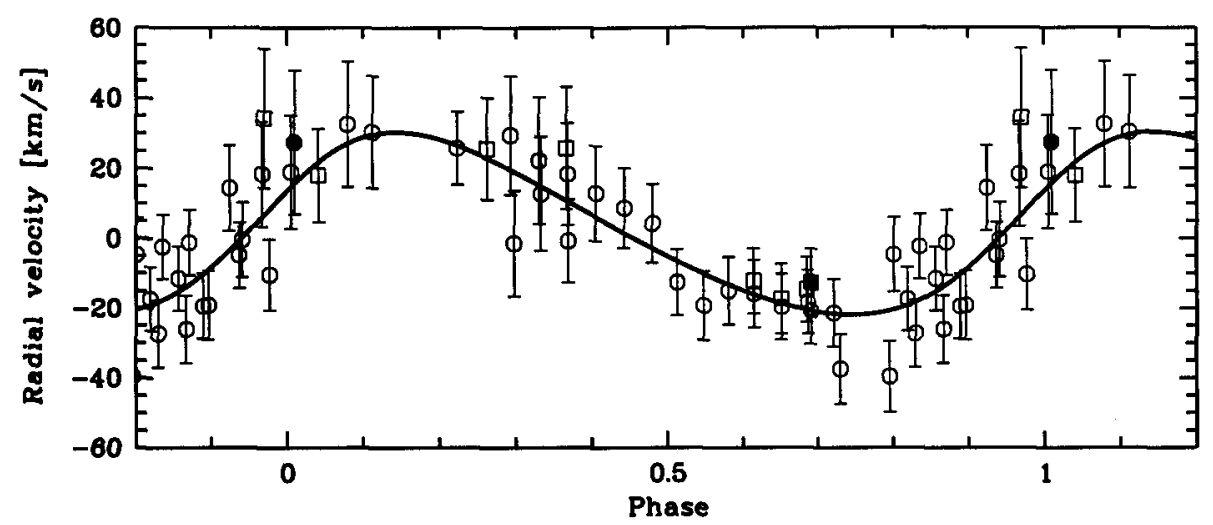

Figure 2. The radial velocity data derived from cross correlation of the He I 4471 line, folded with the orbital period, and the orbital solution (Table 2, VELOC). Data from different observing seasons are marked by different symbols (1990 $\square, 1996 \bullet, 1997$ filled $\square, 1998 \circ$ )

1999, Hummel et al., this volume). Three close similarities between the two stars can be listed: i) In $\phi$ Per, the He I emission component appears only in several lines corresponding to $3^{i} \rightarrow 2^{j}$ transitions. The relative appearance of the line emission in $59 \mathrm{Cyg}$ detected with certainty (HeI 7065, 6678, 5876) is the same as in $\phi$ Per. Trace emission might be present in He I 5015, 3889, but at variance to $\phi$ Per also in HeI 4471. ii) Figs. 1 and 3 show the orbital variations of HeI 6678 emission component. They are in antiphase wrt. the primary RV, but the character of their RV and intensity variations is not consistent with that expected if they were closely connected with orbital motion of the binary components. iii) One of the most distinct features of $\phi$ Per is its He II 4686 emission attributed to a disk around the secondary. Such a feature can be detected in 59 Cyg as well, but in the mean spectrum only. With an intensity of less than $0.5 \%$ above the continuum it is too weak to be found in single HeRos spectra (Fig. 3, right). He II 4686 emission would not be expected if the companion was a Roche-lobe filling giant, but requires a hot star. The orbital parameters are also well consistent with a low mass companion (see Table 2).

Table 2. Orbital elements derived with the VELOC and FOTEL codes

\begin{tabular}{lcc} 
& VELOC & FOTEL \\
\hline$e$ & 0.17 & $0.20 \pm .08$ \\
$\varphi$ & $290^{\circ}$ & $271^{\circ} \pm 35^{\circ}$ \\
$\mathcal{P}[$ fixed] & $28.1702 \pm 0.0014$ day \\
$T_{0}[\mathrm{JD}-24400000]$ & 50021.1 & $50018.9 \pm 2.5$ \\
$K[\mathrm{~km} / \mathrm{s}]$ & 26.1 & $27.2 \pm 8.5$ \\
Mass function & \multicolumn{2}{c}{0.05}
\end{tabular}



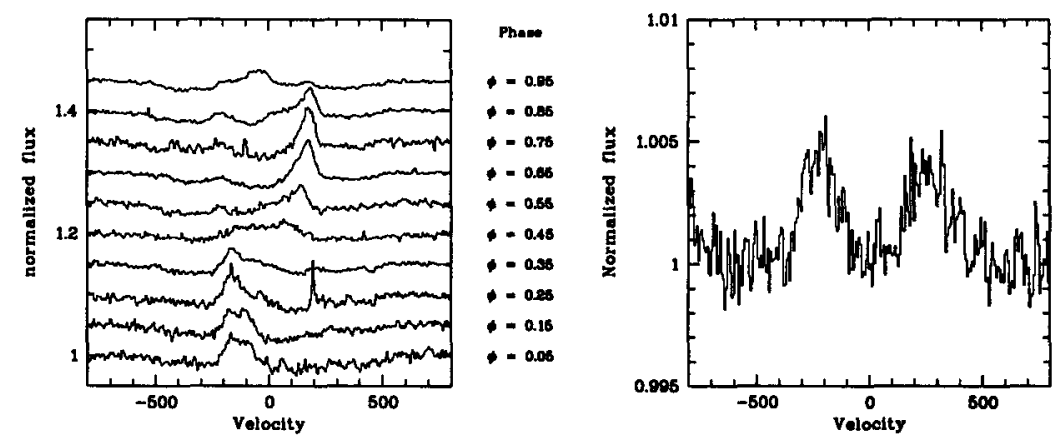

Figure 3. Left: The HeI 6678 emission phased with the orbital period. Phase increases upwards. The similarity to $\phi$ Per (Hummel et al. this volume) is striking. Right: The He II 4686 emission in the mean spectrum

\section{Conclusions}

The binarity of 59 Cyg was confirmed and orbital parameters were derived. The system is viewed at intermediate inclination. Observations indicate several close similarities with $\phi$ Per, which suggest also a similar nature of the secondary companion and mechanism of the He I emission. We assume that the secondary is a hot, compact object illuminating the outer edge of the Be circumprimary disk and possibly having its own weak emission disk. The variable He I emission component might be formed in a sector of the circumprimary disk, illuminated and excited by the secondary hot star. If, however, the companion of one of the best observed objects was not yet recognized as a hot compact source, such systems could exist in abundance, even if only few examples are known. This might have consequences on the possible evolutionary scenarios for Be stars (Gies, this volume).

Acknowledgments. We are grateful to Dr. P. Harmanec for his comments on the orbital solution, and to Drs. W. Schmutz and P. Hadrava for putting their codes at our disposal.

\section{References}

Barker, P.K. 1983, AJ 88, 72;

Doazan, V., Grady, C.A., Snow, T.P., et al. 1985, A\&A 152, 182

Doazan, V., Barylak, M., Rusconi, L., et al. 1989, A\&A 210, 249

Gies, D.R., Willis, C.Y., Penny, L.R., et al. 1993, PASP 105, 281

Hummel, W. 1998, A\&A 330, 243

Kaufer, A. 1998, In: Reviews in Modern Astronomy 11, ed. R. Schielicke, p. 177 Štefl, S., Hummel, W., Rivinius., Th. 1999, A\&A submitted

Tarasov, A.E., Tuominen, I. 1987, Proc. Reg. Eur. IAU Meet., Publ. Astron. Inst. Czechosl. Acad. Sc. 5, 70, p. 127, ed.: P. Harmanec 